\title{
Gas Sensing System Composed of IC-foundry-compatible Microcantilever, Advanced Sensing Material, and Intelligent Signal Processing
}

\author{
Xin Peng, ${ }^{1,2,3}$ Yanqing Lv, ${ }^{1,4}$ Pengcheng Xu, ${ }^{1}$ \\ Xinxin $\mathrm{Li}^{1,2^{*}}$ Haitao $\mathrm{Yu},{ }^{\text {(** }}$ and Jiaqiang $\mathrm{Xu}^{4 * *}$ \\ ${ }^{1}$ State Key Lab of Transducer Technology, Shanghai Institute of Microsystem and Information Technology, \\ Chinese Academy of Sciences, 865 Changning Road, Shanghai 200050, China \\ ${ }^{2}$ School of Information Science and Technology, Shanghai Tech University, \\ 393 Middle Huaxia Road, Shanghai 201210, China \\ ${ }^{3}$ University of Chinese Academy of Sciences, \\ No. 19(A) Yuquan Road, Beijing 100049, China \\ ${ }^{4}$ Department of Chemistry, College of Science, Shanghai University, \\ 99 Shangda Road, Shanghai 200444, China
}

(Received November 4, 2017; accepted February 13, 2018)

Keywords: MEMS, gas sensor, microcantilever, MOF-5, PLL

Gas sensors based on resonant microcantilevers have drawn significant attention in the application of trace molecule detection. In order to realize the practical application of microcantilever-based sensors, three critical points still must be addressed: inexpensive and batch-fabricated microcantilevers, high-performance sensing material and easily operated signal-processing circuit with intelligent software. Herein, a gas sensing system with a multiand-interdisciplinary collaboration mode is proposed. A low-cost and IC-foundry-compatible fabrication technology of the resonant microcantilever is developed. For the example of trace aniline detection, a scalable metal-organic-framework (MOF) material of MOF-5 with ultrahigh surface area and abundant specific aniline-adsorption sites is prepared. For sensing signal processing, an interface circuit with intelligent data processing capability is designed. Composed of the low-cost microcantilever, the advanced MOF-5 sensing material, and the intelligent signal process, the new gas sensing system shows satisfactory performance for trace aniline vapor detection.

\section{Introduction}

Real-time detection of trace gaseous molecules is increasingly in demand in various fields such as industrial production, medical diagnosis, environmental monitoring and antiterrorism. ${ }^{(1-5)}$ In many cases, detection faces formidable challenges because of the low concentration of the target analyte, on-site real-time detection and environmental disturbances. However, most of the commercial analytical devices, such as the gas chromatography-mass spectrometer (GC-

\footnotetext{
*Corresponding author: e-mail: xxli@mail.sim.ac.cn

** Corresponding author: e-mail: yht@mail.sim.ac.cn

${ }^{* * *}$ Corresponding author: e-mail: xujiaqiang@shu.edu.cn

http://dx.doi.org/10.18494/SAM.2018.1798
} 
MS) are rather expensive, bulky, time-consuming and labor-intensive. Therefore, an intelligent sensing system that features ultrafine sensitivity, high selectivity, portability and low cost is urgently demanded. Much effort has been made to explore innovative detection systems to fulfill these application demands. ${ }^{(6-10)}$

With the rapid development of micro-electromechanical systems (MEMS) resonant microcantilever gas sensors have been widely used for chemical molecule detection. ${ }^{(11-17)}$ In addition to high sensitivity and selectivity, resonant microcantilever sensors have the advantages of miniaturization, low-cost batch fabrication, and ability to form an on-chip integrated sensor array. Typically, a resonant microcantilever gas sensor consists of a microcantilever as a physical transducer and a chemically selective sensing layer coated on the microcantilever for specific adsorption of target analyte molecules. The sensing principle is schematically shown in Fig. 1 and described as follows. Once the target analyte molecules are specifically captured by the sensing layer, the increasing mass of the microcantilever will cause a decrease in the resonant frequency of the microcantilever, and then the adsorbed mass can be derived from the change in the resonant frequency. To realize this function, a suitable interface circuit is necessary. Thus, the microcantilever, sensing material, and interface circuit (including the signal processing software) are the key components of the gas sensing system.

Many improvements in resonant microcantilever optimization and gas detection application have been achieved; however, the conventional fabrication of the resonant microcantilevers involves double-sided bulk micromachining of silicon with silicon-on-insulator (SOI) wafers, ${ }^{(18-21)}$ which is not IC-foundry compatible. In addition, both the bulk micromachining and the SOI substrates are very expensive. Moreover, the consistency of the thickness of the fabricated cantilevers is not good, for the etching speed of silicon with deep reactive-ion etching (DRIE) is nonuniform at the wafer level. This will result in poor uniformity of the resonant frequency of the microcantilevers, and further influences the sensitivity consistency. On

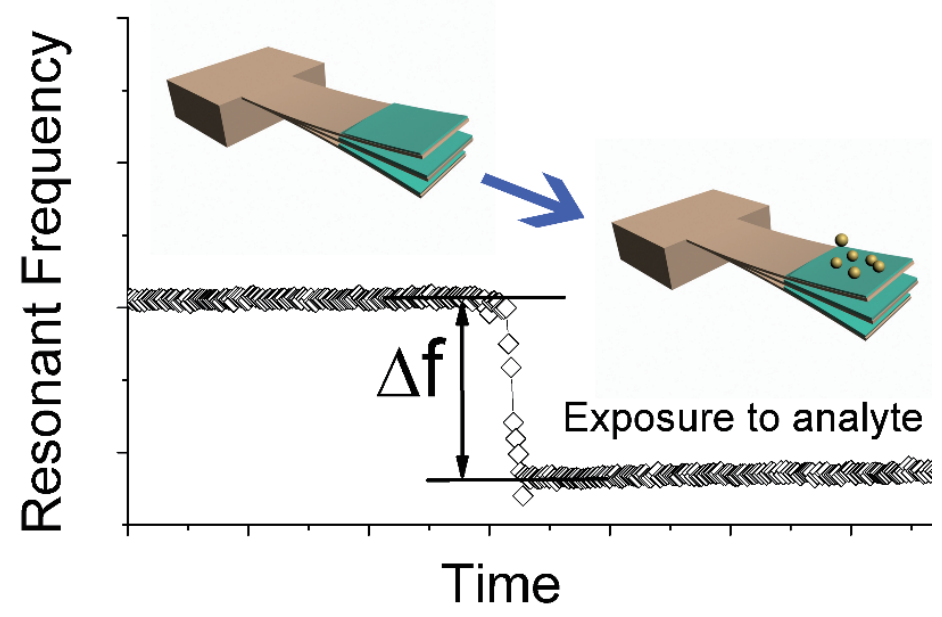

Fig. 1. (Color online) Schematic of the sensing mechanism of resonant microcantilever sensors. 
the basis of the working principle of the microcantilever sensors, we know that the more the target analyte molecules are adsorbed, the larger the resonant frequency shift and the higher the signal-to-noise ratio. Therefore, sensing materials possessing higher specific surface area are preferred for providing more sensing sites to adsorb more gas molecules. In addition, the sensing material should have good selectivity, fast response and repeatability for the target gas. Thus, the choice of the sensing material is also a key point. Another problem that restricts the commercial application of resonant microcantilever sensors lies in the interface circuit and analytic system. Commonly, the major component of the interface circuit is analog phaselocked loops (PLLs). The operation of analog PLLs is complex and requires serval electronic instruments. Particularly when the resonant frequency of the microcantilever drifts too much or a new microcantilever sensor is installed, the phase shifter circuit must be tuned by highly skilled technicians with sophisticated instrumentation to meet the requirements of closed-loop self-excitation. ${ }^{(22,23)}$

To address these issues, collaboration among several institutes/universities is being carried out and a gas sensing system composed of an IC-foundry-compatible microcantilever, advanced sensing material and intelligent signal processing is proposed. A novel IC-foundry-compatible single-sided surface micromachining technique with single-side-polished (111) silicon wafers is proposed and the resonant microcantilevers are batch fabricated using this method at low cost. Advanced material of metal-organic-framework (MOF)-5 with large surface area and good sensing performance to aniline vapor is employed as the sensing material. A frequency tracking system with software PLL is proposed, and the output signal of the sensor is more stable and the noise is ten-fold lower than that of a conventional analog PLL circuit. With the intelligent algorithm, each time the system starts up, the phase difference to be locked is dynamically calculated by advanced signal processing. Even when the sensor was replaced or the environment condition changed, the system could work precisely without adjustment. The sensing experiment is carried out and the sensing system is found to exhibit excellent sensing performance to aniline vapor.

\section{Materials and Methods}

\subsection{Chemicals}

Aniline, 1,4-benzenedicarboxylic acid $\left(\mathrm{H}_{2} \mathrm{BDC}\right)$, zinc acetate, triethylamine and $\mathrm{N}, \mathrm{N}$ dimethylformamide (DMF) were purchased from Sigma-Aldrich. Aniline vapor was purchased from Shanghai SHENKAI Gases Technology Co., Ltd.

\subsection{Synthesis of MOF-5}

The MOF-5 sensing material is synthesized in accordance with the protocol previously described. ${ }^{(24,25)}$ Firstly, $2.5 \mathrm{~g}$ of $\mathrm{H}_{2} \mathrm{BDC}$ and $4.2 \mathrm{~mL}$ of triethylamine are added together into $200 \mathrm{~mL}$ of DMF with stirring to form Stock solution (I). Meanwhile, $8.4 \mathrm{~g}$ of zinc acetate is dissolved completely into $250 \mathrm{~mL}$ of DMF under stirring to form Stock solution (II). Then, 
Stock solution (II) is slowly poured into Stock solution (I) with vigorous stirring. After about $3 \mathrm{hrs}$, the solid precipitates are filtrated and then immersed into $250 \mathrm{~mL}$ of DMF for solvent exchange overnight. Thereafter, the solids are collected and then immersed into $350 \mathrm{~mL}$ of $\mathrm{CHCl}_{3}$ for another $7 \mathrm{~d}$. Then, the $\mathrm{CHCl}_{3}$ solvent is decanted and the solids are obtained and dried at $60{ }^{\circ} \mathrm{C}$ overnight. Before their use in sensing, the MOF-5 products are treated at $150{ }^{\circ} \mathrm{C}$ under vacuum for $6 \mathrm{~h}$.

\subsection{Fabrication of integrated resonant microcantilevers}

Inspired by our recently developed micro-openings inter etch \& sealing (MIS) process, ${ }^{(26)}$ a low-cost fabrication method using single-side-polished (111) silicon wafers for the integrated resonant microcantilevers is developed. The length, width, and thickness of the microcantilever are designed as 200,100 , and $3 \mu \mathrm{m}$, respectively. Thanks to the single-sided fabrication method, the dimension of the microcantilever chip is as small as $1 \times 1 \mathrm{~mm}^{2}$, and the fabrication cost can be controlled below five US cents per chip (6-inch wafer used). The detailed process steps are shown in Fig. 2 and described as follows.

(a) After thermal oxidation, the piezoresistors are formed by boron ion implantation and the

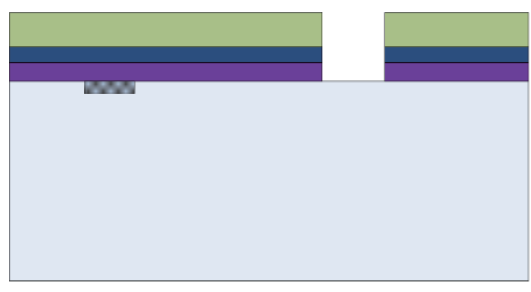

(a)

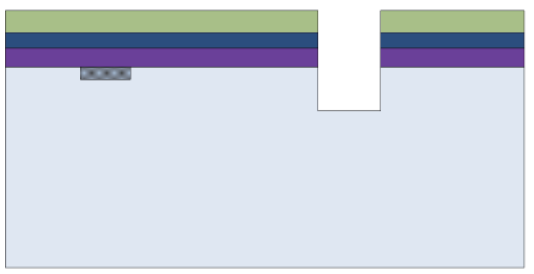

(b)

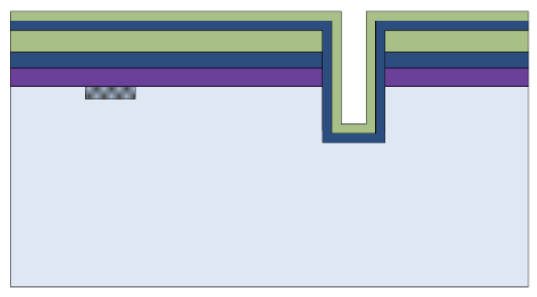

(c)

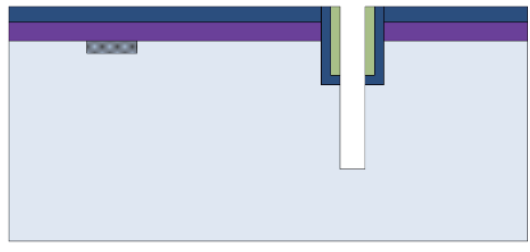

(d)

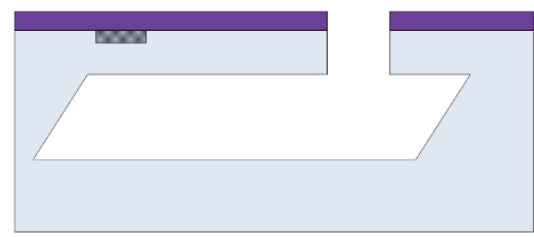

(e)

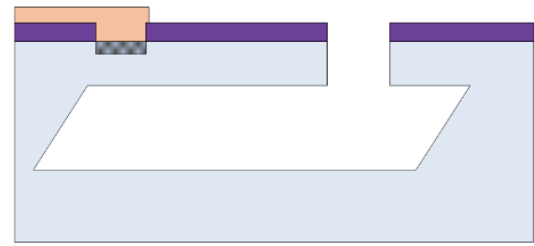

(f)

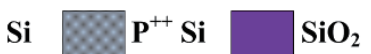

$\mathrm{SiN}_{\mathrm{x}}$

TEOS Al

Fig. 2. (Color online) Fabrication processes of the integrated resonant microcantilever. 
following drive-in process. Then a layer of $\mathrm{SiN}_{x}$ and a layer of tetraethoxysilane (TEOS) are deposited layer by layer onto the $\mathrm{SiO}_{2}$ layer by low-pressure chemical vapor deposition (LPCVD). Next, reactive ion etching (RIE) is used to selectively etch off the three passivation layers.

(b) With the LPCVD-formed passivation layers as the etching mask, silicon DRIE is employed to form the small trench that defines the outline of the microcantilevers.

(c) Another two LPCVD-formed passivation layers of $\mathrm{SiN}_{x}$ and TEOS are also deposited to protect the trench sidewall surface from the following anisotropic wet etch.

(d) RIE is used to selectively etch off the TEOS and $\mathrm{SiN}_{x}$ at the trench bottom to expose bare silicon there, while the passivation layer at the vertical sidewall is retained. After that, silicon DRIE is performed again to deepen the holes.

(e) From the newly exposed bare silicon at the trench sidewall, lateral under etching is started using anisotropic etchant of tetramethylammonium hydroxide (TMAH). According to the anisotropic etching rules for the (111) silicon wafer, ${ }^{(27,28)}$ without protection of the passivation layer, the bottom segment of the trench sidewall is underetched along the lateral directions of $<110>$ and $<211>$, while the top segment of the sidewalls remains unetched owing to protection by the passivation layer. After the TMAH lateral etching is complete, the passivation layers are removed by wet etching.

(f) After the interconnection contact holes are opened with buffered HF, Al thin film is sputtered, patterned, wet etched and sintered to form the interconnection wires. The narrow gap on the edge of the cantilever guarantees that this step can be performed as normal.

\subsection{Characterizations}

The morphologies of the as-synthesized samples are characterized using a field-emission scanning electron microscope (FE-SEM, Hitachi S4800) and a transmission electron microscope (TEM, FEI Tecnai G20).

\subsection{Loading MOF-5 onto microcantilever}

The MOF-5 material $(10 \mathrm{mg}$ ) is added into $1 \mathrm{~mL}$ of deionized water (under ultrasonic) to form a crude suspension that is used as ink in the following material deposition. Then, several drops of the ink are printed onto the top surface near the free end of the microcantilever, using a commercial GIX II Microplotter (Sonoplot Inc.). Thereafter, the microcantilever is dried at 60 ${ }^{\circ} \mathrm{C}$ for about $2 \mathrm{~h}$.

\subsection{Frequency tracking system}

The gas sensing system consists of three main components: the resonant microcantilever sensor, interface circuit, and control center (computer), as illustrated in Fig. 3. The interface circuit is composed of two amplifiers, a filter and a direct digital synthesizer (DDS). The control center commands the DDS to generate a fixed-frequency driving AC signal $\operatorname{Dr}(2 \mathrm{~V}$, 


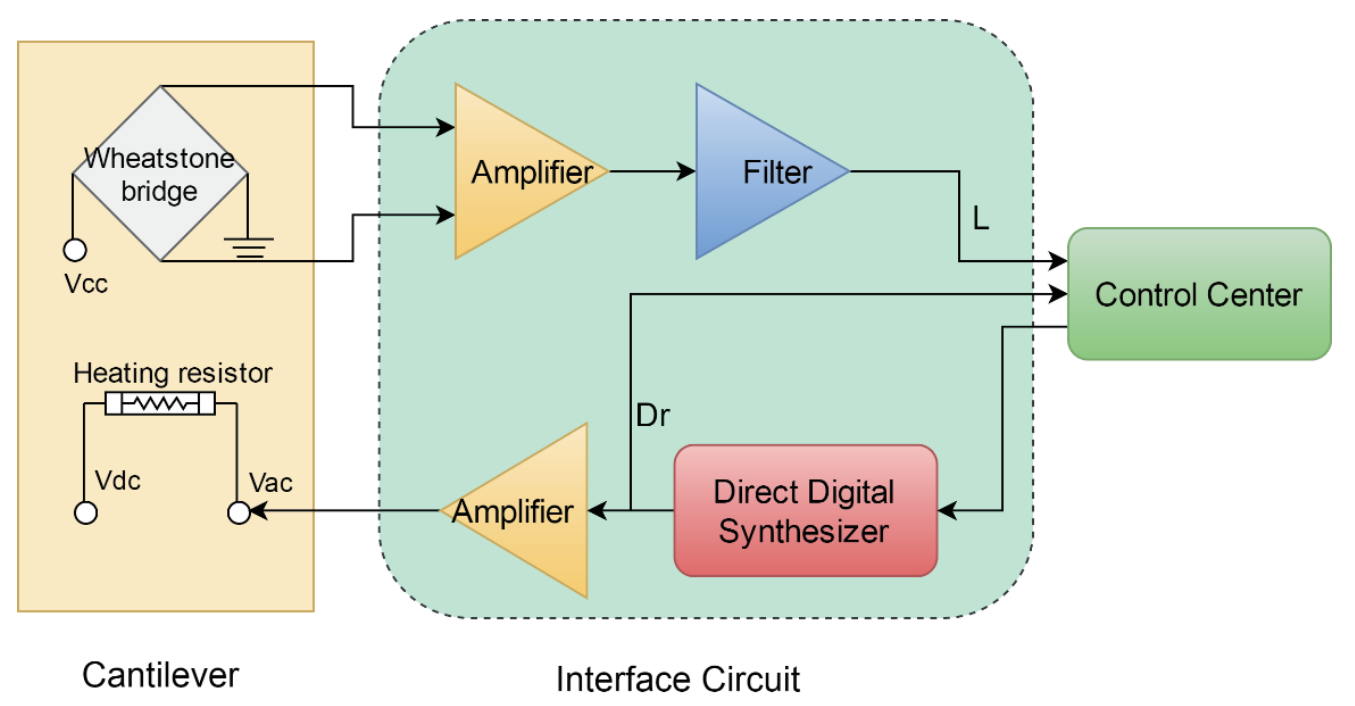

Fig. 3. (Color online) Schematic diagram of the gas sensing system.

peak to peak). This AC signal with a DC bias $(1.8 \mathrm{~V})$ is applied to the heating resistor of the resonant microcantilever to drive the vibration of the microcantilever. The temperature of the cantilever is about $12{ }^{\circ} \mathrm{C}$ higher than the ambient temperature (in case of room temperature) according to the simulation of ANSYS software. The faint signal collected by the piezoresistive Wheatstone bridge will flow through a differential amplifier, and an adjustable filter and then be sampled by the control center as signal $L$.

When the cantilever is working at resonant frequency, the phase difference $\theta_{0}$ between $\mathrm{Dr}$ and $L$ will be a constant. Conventional frequency tracking system of microcantilever sensors employs analog PLL circuit as the interface circuit, which consists of phase detector, operation amplifier, loop filter, phase shifter, voltage controlled oscillator and so on. But there is one crucial problem in that the phase difference between the two input signals of the phase detector is usually fixed in design, while the phase offset of operation amplifier and loop filter change with the frequency of the signal. So, if the sensor is replaced by an alternate one with different resonant frequency, the PLLs would not work properly. ${ }^{(29,30)}$ To solve the problem, a software PLL which consists of the control center and the DDS, is constructed to track the resonant frequency. The control center monitors the frequency of the driving signal $\mathrm{Dr}$ and feedback signal $L$, and calculates the phase difference $\theta$ by the DFT algorithm.

$$
\left\{\begin{array}{c}
u_{x}=\sum_{k=0}^{n-1} u(k) \sin \left(\frac{k}{N} 2 \pi\right) \\
u_{y}=\sum_{k=0}^{n-1} u(k) \cos \left(\frac{k}{N} 2 \pi\right) \\
\varphi=\arctan \left(\frac{u_{x}}{u_{y}}\right) \\
\theta=\varphi_{1}-\varphi_{2}
\end{array}\right.
$$


If $\theta \neq \theta_{0}$, it indicates that the current-driving frequency $f_{D r}$ is not the resonant frequency of the microcantilever. The control center will adjust the frequency of $D r$ to match the resonant frequency and achieve phase locking. The adjustment principle can be described by Eq. (3).

$$
f_{D r \_ \text {new }}=\left\{\begin{array}{l}
f_{D r \_o l d}+\alpha, \theta>\theta_{0} \\
f_{D r \_o l d}-\alpha, \theta<\theta_{0}
\end{array}\right.
$$

Every time the system starts up, the phase difference to be locked is dynamically calculated by advanced signal processing. Even if the sensor was replaced or the environment condition changed, the system could work precisely without adjustment. In addition, with the software PLL, the system can track the resonant frequency faster and with fewer errors than with the analog PLL, which is intricate and easily loses locking. The tracking speed of the system can reach up to $0.1 \mathrm{~s} /$ sample and the noise grade can be controlled under $0.01 \mathrm{~Hz}$.

\subsection{Gas sensing setup}

The fabricated sensor is sealed in a glass testing chamber with a gas inlet and outlet. The frequency tracking system will record the resonant frequency of the microcantilever in real time. Firstly, pure $\mathrm{N}_{2}$ with a fixed flow rate is introduced into the testing chamber and the cantilever sensor vibrates in $\mathrm{N}_{2}$ atmosphere for more than 30 minutes to warm up. Then recording of the resonant frequency of the microcantilever sensor as the base line begins. After a few minutes, aniline vapor diluted to a certain concentration by $\mathrm{N}_{2}$ is introduced into the testing chamber at the same flow rate as that of pure $\mathrm{N}_{2}$ with the help of a four-way valve. The resonant frequency of the microcantilever sensor will decrease owing to the specific adsorption of aniline molecules. When the change of the resonant frequency becomes very slow, which indicates that molecule adsorption is close to the saturation state, the gas passing through the testing chamber is changed back to pure $\mathrm{N}_{2}$. The resonant frequency of the microcantilever sensor will gradually increase to the base line.

\section{Results and Discussion}

The as-prepared MOF-5 material is firstly characterized by SEM. The SEM image in Fig. 4(a) depicts the "sugar-cube-like" morphology of the prepared MOF-5 material. The average side length of the MOF-5 microcubes is approximately $1.5 \mu \mathrm{m}$. The sample is further characterized by TEM. As shown in Figs. 4(b)-4(d), there are many nanopores in the nanocrystals, which indicates that the MOF-5 material has a large specific surface area that is effective for adsorbing more target analyte molecules. With the MOF-5 material loaded on the microcantilever, The SEM image in Fig. 4(e) clearly shows the microcantilever is integrated with a heating resistor for driving vibration and a piezoresistive full Wheatstone bridge for vibration signal output.

Because the wet etching of silicon will stop at (111) crystal surface, the thickness of the asfabricated microcantilevers will have a good consistency in the whole wafer. To verify this 

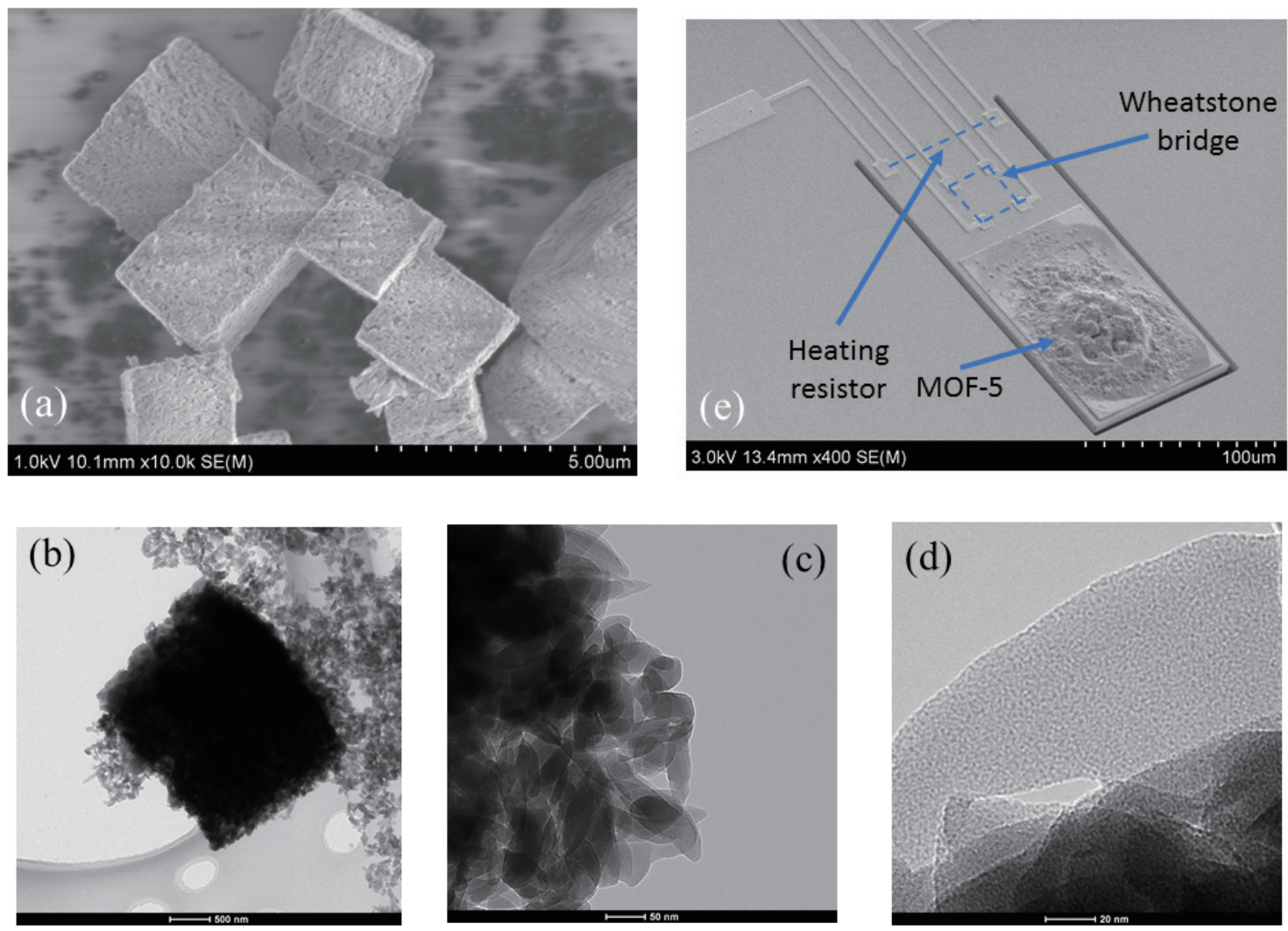

Fig. 4. (Color online) (a) SEM image of the sensing material of MOF-5 nanocubes. (b)-(d) TEM images of the asprepared MOF-5 sample. (e) SEM image of the as-fabricated microcantilever with MOF-5 sensing material loaded.

superiority of the proposed fabrication technique, twenty microcantilevers fabricated in one batch are randomly chosen, and their resonant frequencies are tested by frequency sweeping. The resonant frequency of the microcantilever is relative to the geometric dimensions of the microcantilever including length, width and thickness. Less than $5 \%$ inconsistency of the resonant frequencies has been obtained, which indicates that the fabrication method is highly consistent.

MOF materials are advantageous for use as mass sensing materials owing to their ultrahigh surface area, abundant specific adsorption sites and large volume for molecule capture. Among the diverse MOF materials, MOF-5 has been verified to be a good sensing material for aniline vapor by our previous research. Thus, MOF-5 is chosen as the sensing material to verify the performance of our gas sensing system. Figure 5(a) shows the optical photograph of the interface circuit, while Fig. 5(b) displays the user interface of the system, which exhibits the amplitude-frequency curve, the phrase-frequency curve, the real-time frequency tracking curve of the microcantilever, and some other information. Figure 5(c) shows the room-temperature real-time sensing curve of the sensor to aniline vapor of various concentrations ranging from 3 to $32 \mathrm{ppm}$. The sensing experiments for different concentrations of aniline vapor are performed 


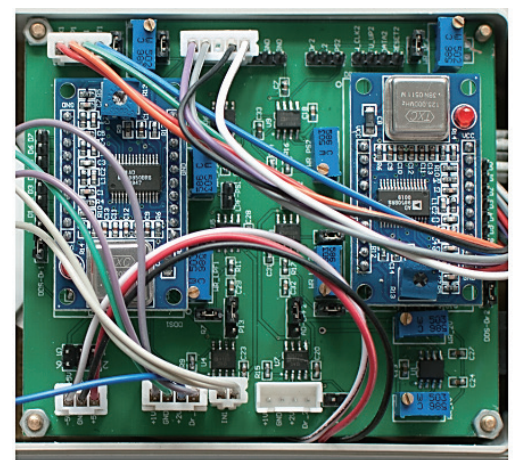

(a)

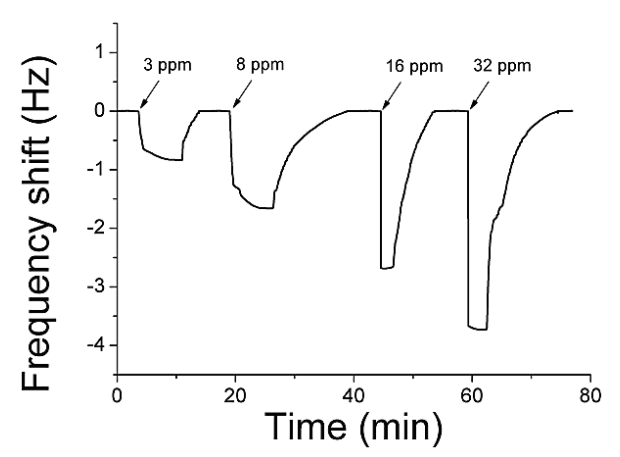

(c)

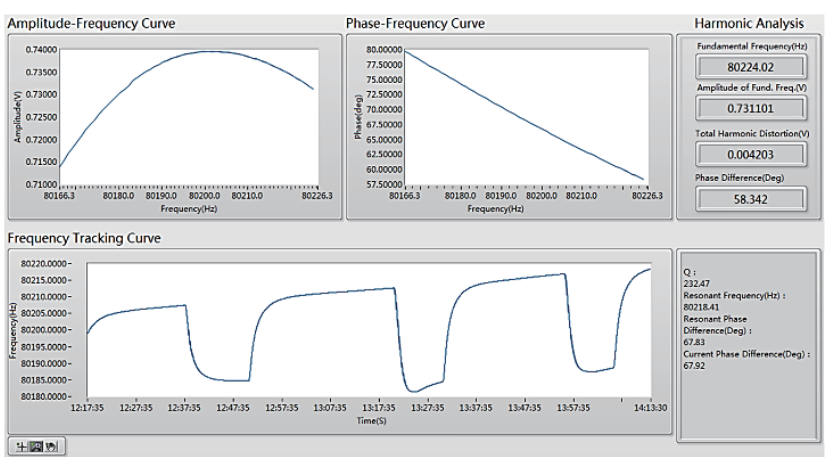

(b)

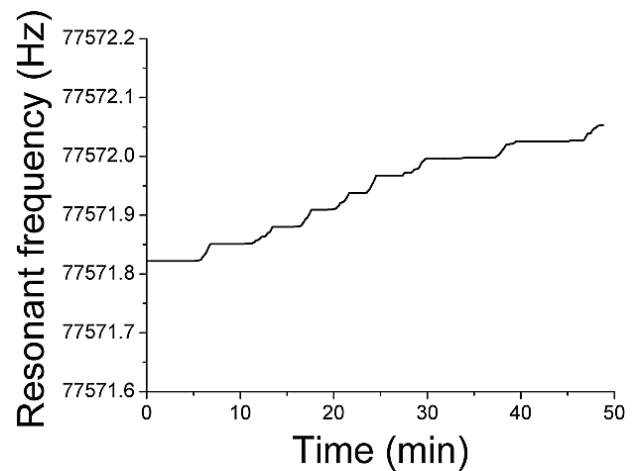

(d)

Fig. 5. (Color online) (a) Interface circuit of the gas sensing system. (b) User interface of the gas sensing system. (c) Responses of the sensor to various concentrations of aniline gas ranging from 3 to $32 \mathrm{ppm}$. (d) Base line of the microcantilever sensor with an ultralow-noise floor.

continuously. Considering that the noise floor of the base line of the output signals is about $\pm 0.05 \mathrm{~Hz}$, as shown in Fig. 5(d), the limit of detection of the sensor to aniline vapor is finer than $300 \mathrm{ppb}$. The results in Fig. 5 verify that our gas sensing system has been fabricated successfully with the proposed multi-and-interdisciplinary collaboration mode.

\section{Conclusions}

A gas sensing system composed of an IC-foundry-compatible microcantilever, advanced MOF-5 sensing material and intelligent signal processing was proposed. By a novel fabrication process, resonant microcantilevers were IC-foundry-compatible batch fabricated at low cost. MOF-5, having a large surface area and good sensing performance to aniline vapor, was employed as the sensing material. With a newly designed interface circuit and intelligent algorithm, the output signal of the sensor was more stable and had a higher signal to noise ratio (SNR). Meanwhile, the operation of the sensing system was more simple and convenient. Sensing experiments were carried out and the sensing system showed a rapid and repeatable response to as little as $3 \mathrm{ppm}$ aniline vapor. Such a high-performance gas sensing system is expected to be widely used in various chemical vapor detection and monitoring applications. 


\section{Acknowledgments}

This research is supported by MOST of China (2016YFA0200800), NSF of China (61571430, 61527818, 61604163), Key Research Program of Frontier Sciences of Chinese Academy of Sciences (QYZDJ-SSW-JSC001). H. T. Yu and P. C. Xu appreciate the financial support of the Youth Innovation Promotion Association CAS (2017278 and 2016213).

\section{References}

1 R. A. Potyrailo: Chem. Rev. 116 (2016) 11877.

2 Y. Suzuki, M. Ye, K. Miyajima, T. Arakawa, S. I. Sawada, H. Kudo, and K. Akiyoshi: Sens. Mater. 27 (2015) 1123.

3 T. L. Li, Y. H. Wu, J. Y. Huang, and S. Q. Zhang: Sens. Actuators, B 243 (2017) 566.

4 S. Yi: Sens. Mater. 29 (2017) 243.

5 S. Khanniche, D. Mathieu, F. Pereira, C. Frenois, D. Colin, C. Barthet, and L. Hairault: Sens. Actuators, B 248 (2017) 470 .

6 C. G. Kuo, J. J. Huang, J. H. Chen, and R. J. Zeng: Sens. Mater. 29 (2017) 533.

7 A. Maity, A. Ghosh, and S.B. Majumder: Sens. Actuators, B 225 (2016) 128.

8 T. Lei, Q. Deng, S.P. Zhang, Z.S. Cai, and C.S. Xie: Sens. Actuators, B 232 (2016) 506.

9 S. Munoz-Aguirre, A. Lopez-Casique, S. Alcantara-Iniesta, J. Castillo-Mixcoat, G. Beltran-Perez, and N. Munoz-Aguirre: Sens. Mater. 26 (2014) 131.

10 L. M. Wei, H. Y. Chen, J. Wang, W. E. Yuang, J. Zhao, D. Xu, and Y. F. Zhang: Sens. Mater. 26 (2014) 9.

11 N. V. Lavrik, M. J. Sepaniak, and P. G. Datskos: Rev. Sci. Instrum. 75 (2004) 2229.

12 A. Boisen, S. Dohn, S. S. Keller, S. Schmid, and M. Tenje: Rep. Prog. Phys. 74 (2011) 036101.

13 H. T. Yu, T. T. Yang, Y. Chen, P. C. Xu, D.-W Lee, and X. X. Li: Anal. Chem. 84 (2012) 6679.

14 H. T. Yu, P. C. Xu, X. Y. Xia, D.-W. Lee, and X. X. Li: IEEE Trans. Ind. Electron. 59 (2012) 4881.

15 Z. Long, L. Kou, M. J. Sepaniak, and X. D. Hou: Rev. Anal. Chem. 32 (2013) 135.

16 P. C. Xu, X. X. Li, H. T. Yu, and T. G. Xu: Sensors 14 (2014) 19023.

17 H. T. Yu, P. C. Xu, D.-W. Lee, and X. X. Li: J. Mater. Chem. A 1 (2013) 4444.

18 H. T. Yu, X. X. Li, X. H. Gan, Y. J. Liu, X. Liu, P. C. Xu, J. G. Li, and M. Liu: J. Micromech. Microeng. 19 (2009) 045023.

19 P. C. Xu, H. T. Yu, and X. X. Li: Anal. Chem. 83 (2011) 3448.

20 A. Hajjam, J. C. Wilson, A. Rahafrooz, and S. Pourkamali: J. Micromech. Microeng. 20 (2010) 125019.

21 F. Yu, H. T. Yu, P. C. Xu, and X. X. Li: J. Micromech. Microeng. 24 (2014) 045005.

22 R. E. Best: Phase-locked Loops: Design, Simulation, and Applications (McGraw-Hill, New York, 2003) Chap. 1.

23 J. R. C. Piqueira and L. H. A. Monteiro: IEEE Trans. Circuits-I 50 (2003) 805.

24 E. Biemmi, S. Christian, N. Stock, and T. Bein: Micropor. Mesopor. Mat. 117 (2009) 111.

25 D. J. Tranchemontagne, J. R. Hunt, and O. M. Yaghi: Tetrahedron 64 (2008) 8553.

26 J. C. Wang and X. X. Li: IEEE Elect. Dev. Lett. 32 (2011) 979.

27 H. Seidel, L. Csepregi, A. Heuberger, and B. Baumgärtel: J. Electrochem. Soc. 137 (1990) 3612.

28 S. W. Lee, S. J. Park, J. P. Kim, S. C. Lee, and D. I. Cho: J. Microelectromech. Syst. 9 (2000) 557.

29 L. H. A. Monteiro, N. C. F. Canto, J. G. Chaui-Berlinck, F. M. Orsatti, and J. R. C. Piqueira: IEEE Trans. Neural Networks 14 (2003) 1572.

30 A. M. Bueno, A. A. Ferreira, and J. R. C. Piqueira: Math. Prob. Eng. 2009 (2009) 266. 